\title{
Highly Enantioselective Organocatalysis with a Mechanically Interlocked Molecule
}

\section{Key words}

mechanically interlocked molecules

catenanes

phosphoric acid catalysis

transfer hydrogenation

tetrahydroquinolines

(S)-4: 82-94\% yield, er from $44: 56$ to $85: 15$ (S) -5 : $86-94 \%$ yield; er from $54.5: 45.5$ to $92: 8$

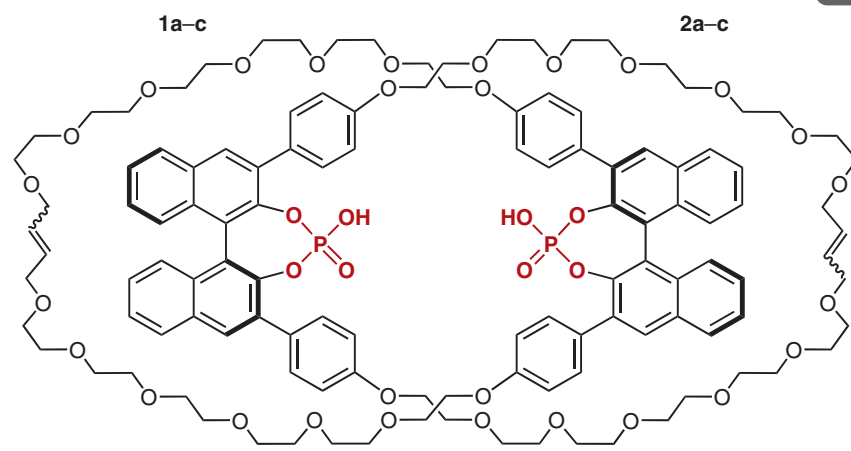

catenated catalyst $(S, S)-\mathbf{3}$ [made up of two units of $(S)-4$ ] Selected examples:

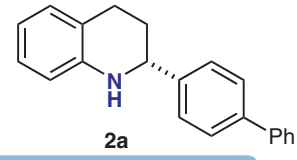

(S,S)-3: $89 \%$ yield; er $=99: 1$ (S)-4: $94 \%$ yield; er $=79.5: 20.5$ (S) $-5: 87 \%$ yield; er $=88: 12$

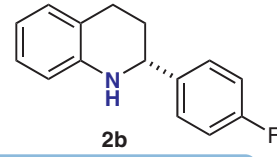

(S,S)-3: $90 \%$ yield; er $=95: 5$

(S)-4: $90 \%$ yield; er $=61.5: 38.5$

(S) -5 : $86 \%$ yield; er $=71: 29$

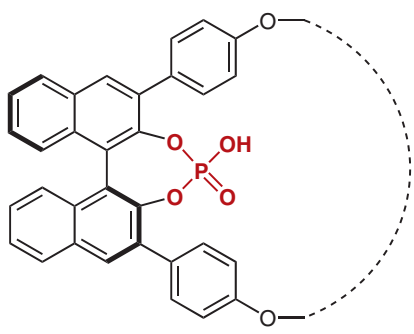

non-interlocked macrocycle $(S)-4$

acyclic catalyst $(S)-5$

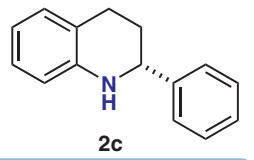

(S,S)-3: $90 \%$ yield; er $=92: 8$

(S) $-4: 82 \%$ yield; er $=44: 56$

(S)-5: $94 \%$ yield; er $=54.5: 45.5$

Proposed transition states (based on DFT calculations):

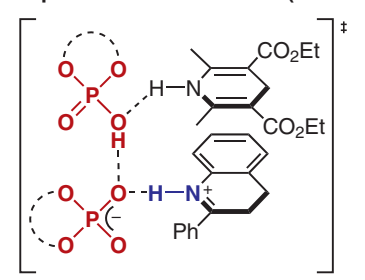

$\Delta \Delta \mathrm{G}^{\ddagger}=3.9$

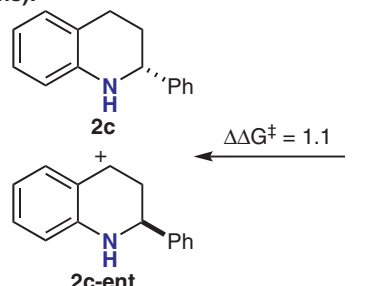

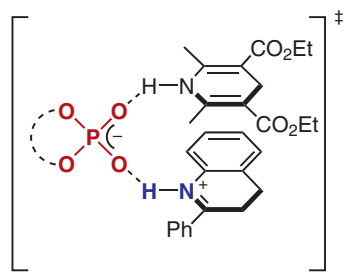

2c-ent

Significance: Niemeyer and co-workers report that the [2]-catenane $(S, S)-\mathbf{3}$, containing two chiral 1,1'-binaphthylphosphoric acids (initial report: Chem. Commun. 2016, 52, 5977), imposes dramatically superior enantioinduction on the transfer hydrogenation of 2-aryl-substituted quinolines, compared with both the corresponding non-interlocked macrocycle (S)-4 and acyclic catalyst (S)-5.

SYNFACTS Contributors: Benjamin List, Jennifer L. Kennemur Synfacts 2017, 13(10), 1085 Published online: 18.09.2017 Dol: 10.1055/s-0036-1591223; Reg-No.: B07717SF
Comment: Computational studies, in which the mechanically interlocked nature of $(S, S)$-3 was mimicked by assuming a simplified non-dissociative dimeric structure, suggest that the interlocked catalyst proceeds through a sandwich-like transition state in the enantiodetermining step. The authors propose that this motif, involving two phosphoric acid moieties bound together in proximity to the reaction site, is responsible for the increase in enantioselectivity. 